\title{
Abstracts
}

Georg Thieme Verlag KG Stuttgart · New York

\section{D-Plant2cells project: Spatial metabolomic, spatial metagenomic, and 3D mass spectrometry imaging, to explore the impact of pesticides on plant metabolome and microbiota}

\author{
LF Nothias \\ ${ }^{1}$ Collaborative Mass Spectrometry Innovative Center, Skaggs School of Pharmacy and Pharmaceutical Sciences, University of California, San Diego, La \\ Jolla, CA 92093, United States \\ 2 Institut de Chimie des Substances Naturelles, CNRS, ICSN UPR 2301, University of Paris-Saclay, Gif-sur-Yvette, 1 avenue de la terrasse, 91198 , \\ France \\ I Protsiuc \\ ${ }^{3}$ European Molecular Biology Laboratory, Meyerhofstrasse 1, 69117 Heidelberg, Germany

\section{T Alexandrov} \\ ${ }^{1}$ Collaborative Mass Spectrometry Innovative Center, Skaggs School of Pharmacy and Pharmaceutical Sciences, University of California, San Diego, La \\ Jolla, CA 92093, United States \\ ${ }^{3}$ European Molecular Biology Laboratory, Meyerhofstrasse 1, 69117 Heidelberg, Germany \\ R Knight \\ ${ }^{4}$ Department of Computer Science and Engineering \\ ${ }^{5}$ Department of Chemistry and Biochemistry, School of Medecine, University of California, San Diego, La Jolla, California 92093, United States
}

\section{A Brunelle}

2 Institut de Chimie des Substances Naturelles, CNRS, ICSN UPR 2301, University of Paris-Saclay, Gif-sur-Yvette, 1 avenue de la terrasse, 91198 , France

\section{Touboul}

2 Institut de Chimie des Substances Naturelles, CNRS, ICSN UPR 2301, University of Paris-Saclay, Gif-sur-Yvette, 1 avenue de la terrasse, 91198 , France

\section{PC Dorrestein}

${ }^{1}$ Collaborative Mass Spectrometry Innovative Center, Skaggs School of Pharmacy and Pharmaceutical Sciences, University of California, San Diego, La Jolla, CA 92093, United States

${ }^{4}$ Department of Computer Science and Engineering

\section{$>$ Author Affiliations}

Further Information

Congress Abstract Full Text (/products/ejournals/html/10.1055/s-0036-1596119)

The "3D-Plant2Cells" project investigates the impact of the agricultural mode on crops grown for human consumption. We are developing an innovative interdisciplinary approach to study the metabolome and the microbiota of plants in threedimension (3D) at three different scales: the whole plant, the fruit, and the cellular scales. The objective of this study is to get a new insight on the metabolome and microbiota response to pesticide treatment. The hypothesis is that the chemistry and microbiome could change upon the use of conventional cultivation managements. To explore this hypothesis, two groups of plants are cultivated in growth chamber and one receives pesticide treatments. The metabolome and the plantassociated microbiota are explored on the whole plant surface (plant scale) by using an innovative interdisciplinary approach [1,2]. Samples are collected on the whole plant and their metabolite content profiled by high-resolution tandemmass spectrometry (HR-MS/MS) and plant-associated microorganism communities by amplicon sequence analysis (metagenomics) [1]. Results of these both inputs are compiled into a bioinformatic model representing a 3D topological map of the metabolome/microbiome over the entire organism surface [1]. Mass spectrometry data will be further interpreted using MS/MS molecular networking [1]. In the meantime, we are studying fruits of the cultivated specimens by 3D mass spectrometry imaging ${ }^{3}$ to study the metabolome/microbiota response to pesticide treatments along with the pesticide distribution in 3D. The fruit and cell scale are explored by analyzing serial sections using MALDI, nanoDESI, 3DTOF-SIMS coupled to HR-MS/MS. The presentation will aim at introducing the project, and to highlight some methodological approaches used to study the microbiota-chemistry relationships in species or ecosystem.

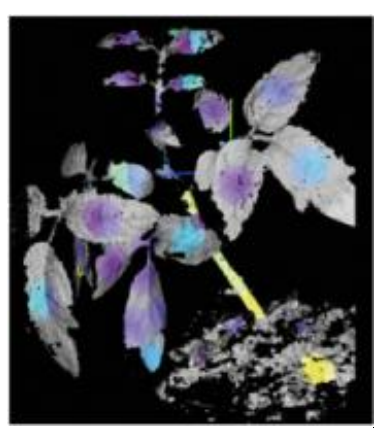


Figure 1. Spatial distribution of metabolites on a tomato plant 3D model. Data derived from LC-HR-MS/MS.

Acknowledgement: The 3D-Plant2Cells project is funded by the European Union under the Horizon 2020 framework programme for research and innovation (Marie Sklodowska-Curie Actions, individual global fellowship, MSCA-IF-2016, 704786).

Keywords: spatial metabolomic, spatial metagenomic, 3D mass spectrometry imaging, molecular networking, plantassociated microbiota, pesticide.

\section{References:}

[1] Bouslimani A, Porto C, Rath CM, Wang M, Guo Y, Gonzalez A, Berg-Lyon D, Ackermann G, Christensen GJM, Nakatsuji T, Zhang L, Borkowski AW, Meehan MJ, Dorrestein K, Gallo RL, Bandeira N, Knight R, Alexandrov T, Dorrestein PC. Molecular cartography of the human skin surface in 3D. Proc Natl Acad Sci 2015; 112: $2120-2129$

[2] Palmer AD, Alexandrov T. Imaging mass spectrometry at its tipping point. Anal Chem 2015; $87: 4055-4062$

[3] Touboul D, Brunelle A. What TOF-SIMS can bring more than other MS imaging methods?. Bioanalysis 2016; in press, doi: $10.4155 /$ bio.16.11.

(c) 2019 Georg Thieme Verlag KG I Imprint (https://www.thieme.de/de/thieme-gruppe/Imprint-

Thieme-EN.htm). I Privacy_policy statement (https://www.thieme.de/de/thieme-gruppe/privacy-

policy-statement-Thieme-EN.htm). I Smartphone Version (Lproducts/ejournals/abstract/10.1055/s-

0036-1596119?device $=$ mobile 